\title{
Condiciones de Salud Oral y Estado Protésico de la Población Adulta Mayor Atendida en la Red Hospitalaria Pública de Medellín (Colombia)
}

\author{
Oral Health Conditions and Prosthetic Status in Elderly Population \\ Assisted by the Public Hospital Network of Medellin (Colombia)
}

Adriana Posada-López", ; Andrés A. Agudelo-Suárez \& Edwin J. Meneses-Gómez**

POSADA-LÓPEZ, A.; AGUDELO-SUÁREZ, A. A. \& MENESES-GÓMEZ, E. J. Condiciones de salud oral y estado protésico de la población adulta mayor atendida en la red hospitalaria pública de Medellín (Colombia). Int. J. Odontostomat., 10(1):161$171,2016$.

RESUMEN: Este estudio pretendió analizar las condiciones de salud oral y el estado protésico de la población adulta mayor atendida en la red hospitalaria pública de Medellín (Colombia). Se realizó un estudio transversal mediante muestreo bi-etápico en las unidades hospitalarias y centros de salud adscritos a esta red. Participaron 342 adultos de 65 y más años (58,2 \% mujeres). Se recogió información a través de encuesta y examen clínico. Variables: Sociodemográficas: edad, sexo, estrato socioeconómico, nivel educativo, zona de residencia, apoyo social (Duke-11). Salud oral: Percepción de salud oral, presencia de problemas orales en el último mes. Indicadores clínicos: Índice COPD, dientes presentes, estado de prótesis dentales, problemas de la mucosa oral. Análisis univariado y bivariado y cálculo de pruebas Chi cuadrado para observar diferencias estadísticamente significativas entre las variables. Se encontró que las personas $\geq 75$ años, los hombres, la población de estratos socioeconómicos y nivel educativo bajos, ubicados en área urbana y con apoyo social bajo tienden a reportar mala salud oral autopercibida. El promedio de dientes presentes en la población fue de $5,7( \pm 7,8)$, lo que implica un COPD promedio de $24,2( \pm 5,9)$, con diferencias de acuerdo a variables sociodemográficas. Más del $55 \%$ de los hombres y las mujeres requieren cambio de prótesis inferior y un $70 \%$ requieren cambio de prótesis superior. Se encontraron problemas de la mucosa oral: candidiasis (12\%), ulceras (7\%), leucoplasia (7\%), liquen plano (1\%). En conclusión se evidencian deficientes condiciones de salud oral que pueden ser explicadas por la situación de vulnerabilidad social que enfrenta esta población y barreras de acceso a servicios de salud oral preventivos y curativos.

PALABRAS CLAVE: salud oral, prótesis dental, higiene oral, salud de la persona mayor, atención odontológica.

\section{INTRODUCCIÓN}

Las enfermedades orales han sido consideradas como problemas de salud pública por su magnitud y severidad (Petersen, 2003; Petersen et al., 2005). Si bien estas patologías no constituyen un riesgo alto de muerte en comparación con otras condiciones, si representan costos económicos, días de incapacidad y menoscabo de la calidad de vida por dolor, pérdida dental, dificultades psicológicas y costos por tratamientos de rehabilitación parcial o total de las estructuras dentarias (Sheiham, 2005). En la epidemiología de estas condiciones intervienen factores biológicos propios del individuo, como también determinantes relacionados con el contexto social y político y aquellos relacionados con el acceso a los servicios de salud oral para los diferentes grupos sociales (Watt, 2007). Informes y reportes institucionales dan cuenta como la situación de salud oral en indicadores como la caries dental difieren sustancialmente entre los países dependiendo de su nivel de renta y desarrollo social (Petersen; Petersen et al.).

\footnotetext{
Facultad de Odontología, Universidad de Antioquia, Medellín, Colombia.

* Facultad de Odontología, Fundación Universitaria Autónoma de las Américas, Medellín, Colombia.

Financiado por: E.S.E Metrosalud. Medellín (Código: C02-E11-L3-01).
} 
Por otro lado, asumir una perspectiva de ciclo vital en el análisis epidemiológico oral implica reconocer las particularidades de los grupos etáreos con el fin de establecer prioridades y estrategias acordes con la realidad social y las necesidades de los individuos (Organización Mundial de la Salud et al., 2002). Es así que una de las poblaciones que se ha constituido como un segmento significativo en la sociedad tanto en términos demográficos, como por su exposición a situaciones de vulnerabilidad social es la población adulta mayor. Según el reporte del Population Reference Bureau (2014), la población de 65 años y más representa el $7 \%$ de la población mundial. Los datos para Colombia son más o menos similares y se tiene que la esperanza de vida ha aumentado de 61 años en 1970 a 75 años en el 2013 (Population Reference Bureau).

Concretamente en Colombia, la situación de vulnerabilidad de esta población se traduce en el aumento de enfermedades crónicas y degenerativas, problemas de salud mental, sentimiento de soledad (Profamilia, 2010; Cardona Jiménez et al., 2013; Estrada Restrepo et al., 2013). En cuanto a la salud oral, los reportes del último Estudio Nacional de Salud Bucal -ENSAB IV-, realizado entre 2013 y 2014 dan cuenta de que la prevalencia de caries dental en la población de 65 a 74 años es del $43,4 \%$. En este mismo grupo el $92 \%$ ha perdido dientes por caries y una buena parte son edentados totales (Ministerio de Salud y Protección Social, 2013-2014). Esta situación representa numerosos desafíos políticos y sociales para disminuir las desigualdades e inequidades en salud.

En el colectivo de los adultos mayores el estado de las prótesis dentarias, la satisfacción que tienen las personas con estas y la higiene, afectan directamente sus tejidos blandos, dado que son estos los que soportan la presencia de dicho aditamento e indirectamente inciden en la salud del sistema estomatognático y la salud general. Como consecuencia de unas prótesis dentales inadaptadas, el estado nutricional puede verse afectado por una masticación ineficiente, ocasionando problemas de alimentación. La presencia de dolor es otro aspecto relacionado con el desarrollo normal de la función masticatoria, en este caso la articulación temporomandibular podría estar involucrada. Otros aspectos relevantes a tener en cuenta son la dimensión social y psicológica, ya que en una buena parte de estos adultos al no tener una prótesis funcional, ven menoscabadas sus relaciones con los demás; ya que al afectarse la fonación se impide una adecuada comunicación lo cual tiene consecuencias en la autoestima de las personas (Misrachi et al., 2002; Cardentey García et al., 2011).
Colombia ha tenido como políticas relacionadas con salud oral el Plan Nacional de Salud Bucal -PNSBadoptado por Resolución 3577 de 2006 (Ministerio de la Protección Social, 2006), el Plan Nacional de Salud Pública 2007-2010, mediante el Decreto 3039 de 2007 (Ministerio de la Protección Social 2007), y más actualmente, el Plan Decenal de Salud Pública 2012- 2021, donde la salud oral se incorpora al componente de enfermedades crónicas y aquellas relacionadas con estilos de vida (Ministerio de Salud y Protección Social 2013). A nivel global, la Organización Mundial de la Salud ha establecido unas metas en salud oral para el año 2020, que no son específicas puesto que deben ser adaptadas a los contextos de cada país, de acuerdo a sus necesidades y estrategias locales e involucran aspectos como por ejemplo el número de dientes presentes a edades trazadoras en el curso de vida, y la prevalencia de caries dental y otras condiciones orales en grupos poblacionales específicos por edad (Hobdell et al., 2003).

Los adultos mayores actualmente ocupan un espacio cada vez más importante en la sociedad, pero en términos generales la utilización de los servicios odontológicos en esta población es menor en comparación con los servicios médicos. Esto se debe entre otros factores a la falta de políticas en salud oral y estrategias para esta población, la priorización de otros grupos etáreos para dar atención odontológica integral preventiva y curativa y otras barreras de acceso a los servicios de salud oral (Agudelo-Suárez et al., 2014). Se hace necesario por lo tanto que se sensibilicen las diferentes instancias en la sociedad con el fin de conocer las reales condiciones de salud oral de la población adulta mayor en contextos locales específicos, identificar situaciones de desigualdad e inequidad, y dar respuesta efectiva a las necesidades de la población en forma oportuna y eficiente.

En este contexto el objetivo de este estudio fue analizar las condiciones de salud oral y el estado protésico de la población adulta mayor atendida en la red hospitalaria pública de Medellín (Colombia).

\section{MATERIAL Y MÉTODO}

Se trata de un estudio transversal. La población de estudio la constituyeron los adultos mayores de la red hospitalaria pública de Medellín -E.S.E METROSALUD- (Empresa Social del Estado (E.S.E) Metrosalud) que acuden a los servicios de consulta ex- 
terna en su red de servicios de Medellín. Se tuvieron en cuenta para este estudio a hombres y mujeres con $65 \mathrm{y}$ más años y que fueron seleccionados a partir de los registros de consulta de primera vez a cargo de la unidad estadística de la institución para el año 2011. Los criterios de exclusión fueron: discapacidad motora que le impidiera al sujeto desplazarse a su centro de atención, discapacidad cognitiva y/o mental expresada en el contacto telefónico.

Se determinó una muestra inicial de 352 adultos mayores mediante la aplicación de un muestreo en dos etapas: En primer lugar, muestreo aleatorio simple para la selección de Unidades Hospitalarias - UH- y Centros de Salud -CS- en toda la ciudad de Medellín. El segundo, un muestreo por cuotas, proporcional al número de adultos mayores asignados para cada UH y CS y seleccionados aleatoriamente. Para el cálculo del tamaño muestral se tuvo en cuenta la prevalencia del 29,1 $\%$ de un nivel de calidad de vida bajo, demostrada por otros estudios (Montero et al., 2011); un nivel de confianza del $95 \%$ y un error de muestreo del $5 \%$, más un sobremuestreo del $10 \%$ por posibles pérdidas de información. Sin embargo, para este análisis, se utilizó la información de 342 adultos mayores (58,2 \% mujeres), lo cual constituye un $97 \%$ de la muestra total luego de hacer depuración de los datos.

El proyecto más amplio contempló la utilización de encuesta estructurada y examen odontológico completo, los cuales fueron realizadas por un grupo de trabajo de campo (2 examinadores y 4 encuestadores), los cuales fueron capacitados y calibrados para obtener encuestas de calidad. Se realizó una prueba piloto en 10 adultos mayores con el fin de corregir revisar el lenguaje, la adaptación cultural de las preguntas y la consistencia general de los instrumentos de recogida de información. El trabajo de campo se realizó entre los meses de marzo y diciembre de 2013. Los estándares del examen oral siguieron los parámetros definidos en los métodos básicos de la Organización Mundial de la Salud -OMS- para realización de encuestas de salud bucodental en su $4^{a}$ versión (World Health Organization, 1997).

Concretamente para este estudio se consideraron las siguientes variables: 1) Sociodemográficas: tipo de centro, edad, sexo, estrato socioeconómico, nivel educativo, zona de residencia, apoyo social, ocupación (esta fue obtenida de la clasificación internacional de ocupaciones adaptada para Colombia (Departamento Administrativo Nacional de Estadística (DANE), 2005), y se categorizó en no manual -directivas, profesionales universitarios, científicos e intelectuales, técnicos, postsecundarios no universitarios y asistentes, empleados de oficina, trabajadores de los servicios y vendedores o comerciantes, manual -agricultura, manuales de la industria y de la construcción y trabajadores no calificados-, labores del hogar y otras -jubilados y estudiantes-; 2) Indicadores de salud oral: índice de dientes cariados, obturados y perdidos por caries -COPD- de Klein y Palmer (World Health Organization), dientes presentes, estado de salud oral autopercibido (bueno/malo), satisfacción estado dental (satisfecho/insatisfecho), presencia de problemas orales en los últimos 30 días (si/ no); 3) Estado protésico: presencia y tipos de prótesis superior e inferior, necesidad de cambio de prótesis superior y/o inferior (si/no), remoción de la prótesis superior o inferior (si/no), limpieza de mucosa (si/no). 3) Lesiones de la mucosa oral (si/no): leucoplasia, liquen plano, ulcera: aftosa, herpética, traumática, candidiasis, absceso y otros trastornos.

En un primer momento se realizó análisis del índice COPD y sus diferentes componentes (cariados, obturados y perdidos) y del número de dientes presentes según las variables sociodemográficas y pruebas Chi cuadrado para observar diferencias en los promedios (t de student). Posteriormente se calculó la prevalencia de los indicadores de salud con su respectivo intervalo de confianza al $95 \%$ (IC95\%), de acuerdo a las variables sociodemográficas. En tercer lugar se realizó análisis separadamente para hombres y mujeres según las variables de estado protésico y para la presencia de lesiones de la mucosa oral y pruebas Chi cuadrado para observar diferencias significativas en las distribuciones porcentuales.

Esta investigación fue acorde con la normativa internacional -Declaración de Helsinki- (World Medical Association General Assembly, 2004) y nacional -Resolución 008430, 1993, Ministerio de Salud- (República de Colombia. Ministerio de Salud y Protección Social, 1993). Se garantizó la confidencialidad durante todo el proceso de investigación y la población participante en el estudio dio su consentimiento oral y escrito para participar en la investigación. Teniendo en cuenta que la población adulta mayor es sensible, a todos los participantes y sus familiares se les informó sobre los objetivos del estudio y se les comunicó que podían retirarse libremente y sin ningún perjuicio en cualquier momento si lo deseaban. Adicionalmente recibieron información sobre los tratamientos que requerían y donde debían acudir. El protocolo de investigación fue aprobado por el Comité de Ética de la E.S.E Metrosalud (acta 09-2011). 


\section{RESULTADOS}

En la Tabla I se muestran los indicadores de dientes presentes y el índice COPD y sus componentes de acuerdo a variables sociodemográficas. El promedio de dientes presentes es mayor en los menores de 75 años, en personas de estrato medio, en personas de mayor nivel educativo (se encontraron gradientes y diferencias estadísticamente significativas, $p<0,01)$. De igual forma el promedio fue mayor en personas del área urbana y de ocupaciones no manuales. Con relación a los dientes cariados, llama la atención que se encontraron diferencias estadísticamente significativas para las variables: tipo de centro (mayor promedio en aquellos atendidos en Unidad Hospitalaria, $p<0,01$ ), y sexo (mayor promedio en hombres, $p<0,01$ ). En cuanto a los dientes obturados se encontraron diferencias estadísticamente significativas por sexo (mayor promedio en mujeres, $\quad p<0,01)$, por estrato socioeconómico (mayor promedio en estratos medios, $p<0,01$ ), por nivel educativo (mayor en secundaria-universitaria) y ocupación (mayor en ocupaciones manuales). El promedio de dientes perdidos es mayor en personas sin estudios $(p<0,01)$ y en labores del hogar $(p<0,01)$. Por último el COPD es de 24,2 $( \pm 5,9)$, con diferencias según factores sociodemográficos y estadísticamente significativas según nivel educativo (mayor en sin estudios), edad (mayor promedio en personas $\geq 75$ años), zona (mayor en área rural), ocupación (mayor en otras labores).

En cuanto a los indicadores de salud oral autopercibida (Tabla II), las personas atendidas en centros de salud, los hombres, la población $\geq 75$ años, con estudios primarios, en zona urbana, con apoyo social bajo $(p<0,01)$ y en otras ocupaciones $(p<0,01)$ reportaron su estado de salud como malo. Sin embargo cuando se analiza la satisfacción con el estado dental, las personas más insatisfechas son las mujeres, los menores de

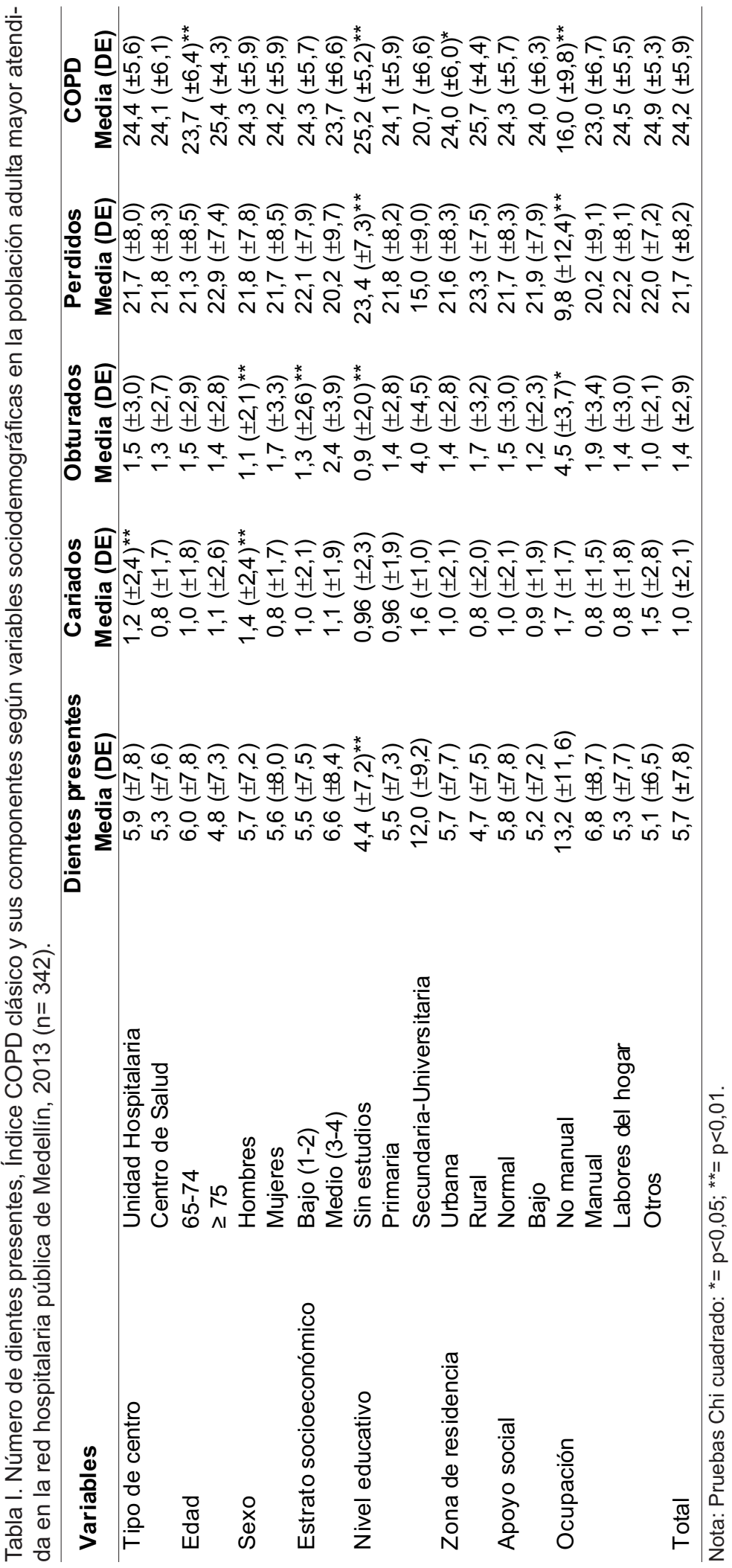




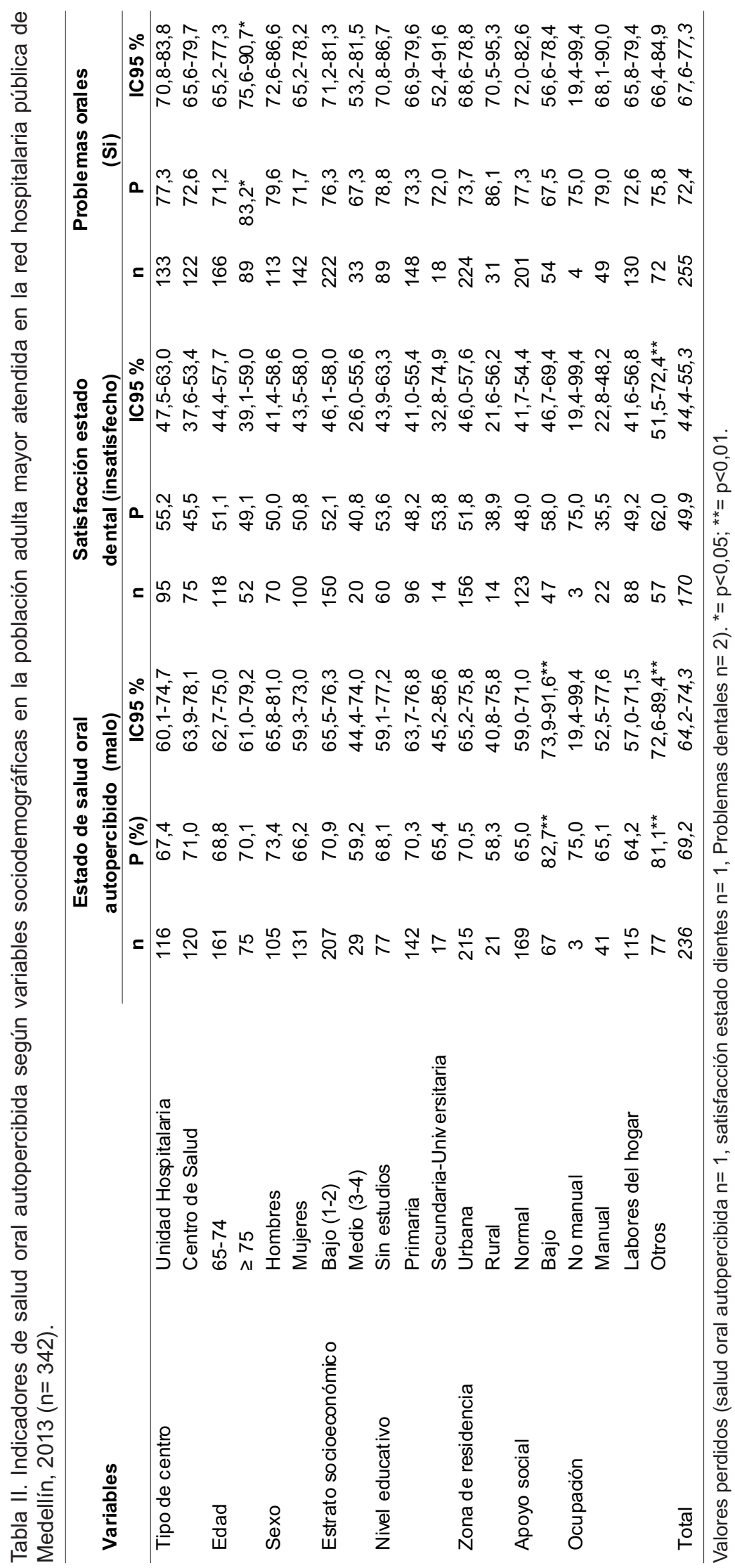

75 años, los atendidos en Unidad Hospitalaria. En cuanto a la ocupación los que se dedican a otras labores son mas insatisfechos $(p<0,01)$. Una cuarta parte de la población encuestada reporta problemas orales en el último mes, con diferencias según factores sociodemográficos, siendo estadísticamente significativas para el caso de la variable edad (mayor reporte en población $\geq 75$ años, $p<0,05)$.

La Tabla III muestra las diferentes variables analizadas en el estado protésico de las personas examinadas en el estudio. Un $68 \%$ de los hombres y $72 \%$ de las mujeres necesitan cambio en la prótesis superior y un poco más de la mitad en ambos sexos necesitan cambio en la prótesis inferior. En cuanto a las condiciones de higiene oral, el $74 \%$ de los hombres y el $64 \%$ mujeres no se retiran la prótesis superior en la noche para dormir. El $81 \%$ de los hombres y $48 \%$ de las mujeres no se retiran la prótesis inferior para dormir con diferencias estadísticamente significativas $(p<0,001)$. Una tercera parte de los hombres y casi una quinta parte de las mujeres no se limpian la mucosa oral superior $(p<0,05)$, con porcentajes similares en el caso de la mucosa oral inferior y diferencias estadísticamente significativas $(p<0,01)$.

Por ultimo, teniendo en cuenta las lesiones de la mucosa oral (Fig. 1), los hombres presentaron mayor prevalencia de candidiasis $(12 \%)$ y leucoplasia y las mujeres presentaron mayor prevalencia de ulceras $(6,5 \%)$ y candidiasis $(6,5$ $\%)$. Se presentaron diferencias estadísticamente significativas entre hombres y mujeres $(p<0,001)$ para el caso de las lesiones leucoplásicas. 
POSADA-LÓPEZ, A.; AGUDELo-SUÁREZ, A. A. \& MENESES-GómEZ, E. J. Condiciones de salud oral y estado protésico de la población adulta mayor atendida en la red hospitalaria pública de Medellín (Colombia). Int. J. Odontostomat., 10(1):161-171, 2016.

Tabla III. Estado protésico de la población adulta mayor atendida en la red hospitalaria pública de Medellín, $2013(n=342)$.

\begin{tabular}{|c|c|c|c|c|}
\hline \multicolumn{2}{|l|}{ Estado protésico } & \multirow[t]{2}{*}{$\begin{array}{r}\text { Hombres } \\
(n=143)\end{array}$} & \multirow[t]{2}{*}{$\begin{array}{l}\text { Mujeres } \\
(n=199)\end{array}$} & \multirow[t]{2}{*}{ Valor $\mathbf{P}^{*}$} \\
\hline Presencia & Superior & & & \\
\hline \multirow[t]{10}{*}{ de prótesis } & No prótesis & 30,1 & 18,1 & \multirow{5}{*}{0,022} \\
\hline & Prótesis fija & 1,4 & 4,5 & \\
\hline & Prótesis removible & 14,0 & 11,6 & \\
\hline & Ambas, prótesis fija y removible & 0,7 & 0,0 & \\
\hline & $\begin{array}{l}\text { Prótesis total } \\
\text { Inferior }\end{array}$ & 53,8 & 65,8 & \\
\hline & No prótesis & 63,6 & 46,7 & \multirow{5}{*}{0,035} \\
\hline & Prótesis fija & 0,0 & 1,5 & \\
\hline & Prótesis removible & 7,7 & 9,5 & \\
\hline & Ambas, prótesis fija y removible & 0 & 1 & \\
\hline & Prótesis total & 28,7 & 41,2 & \\
\hline Necesidad o & Superior & & & \multirow{4}{*}{0,422} \\
\hline \multirow[t]{5}{*}{ cambio de prótesis } & No & 32,2 & 28,1 & \\
\hline & $\mathrm{Si}$ & 67,8 & 71,9 & \\
\hline & Inferior & & & \\
\hline & No & 44,8 & 40,7 & \multirow{2}{*}{0,455} \\
\hline & $\mathrm{Si}$ & 55,2 & 59,3 & \\
\hline \multirow{6}{*}{$\begin{array}{l}\text { Remoción } \\
\text { de prótesis }\end{array}$} & Superior & $(n=77)$ & $(n=131)$ & \multirow{4}{*}{0,140} \\
\hline & No & 74,0 & 64,1 & \\
\hline & $\mathrm{Si}$ & 26,0 & 35,9 & \\
\hline & Inferior & $(n=41)$ & $(n=82)$ & \\
\hline & No & 80,5 & 47,6 & \multirow{2}{*}{$<0,001$} \\
\hline & $\mathrm{Si}$ & 19,5 & 52,4 & \\
\hline Limpieza & Superior & $(n=77)$ & $(n=131)$ & \multirow{4}{*}{0,012} \\
\hline \multirow[t]{5}{*}{ de la mucosa } & No & 33,8 & 18,3 & \\
\hline & $\mathrm{Si}$ & 66,2 & 81,7 & \\
\hline & Inferior & $(n=41)$ & $(n=82)$ & \\
\hline & No & 36,6 & 18,3 & \multirow{2}{*}{0,026} \\
\hline & $\mathrm{Si}$ & 63,4 & 81,7 & \\
\hline
\end{tabular}

${ }^{*}$ Pruebas Chi cuadrado de distribución de frecuencias.

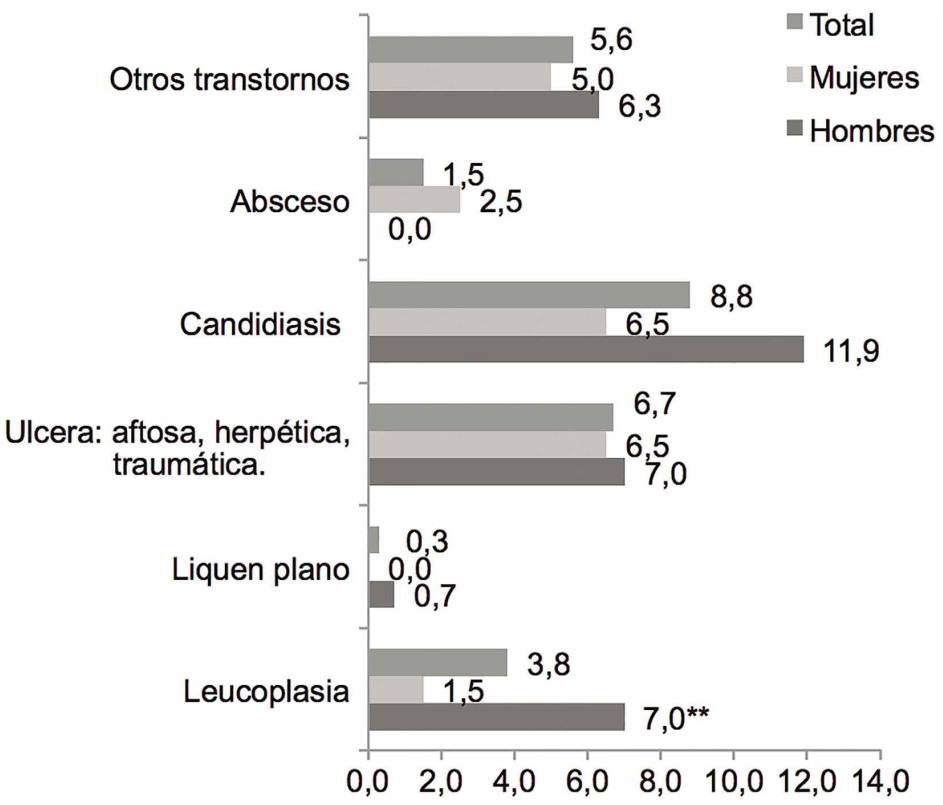

Fig. 1. Prevalencia de lesiones de la mucosa oral en la población adulta mayor atendida en la red hospitalaria pública de Medellín, $2013(n=342)^{*}$. Nota: Prueba Chi cuadrado de diferencia entre proporciones, ${ }^{* *}=$ $p<0,01$. 


\section{DISCUSIÓN}

Los hallazgos principales de este estudio, dan cuenta de los factores relacionados con la magnitud de los indicadores de salud oral analizados. En términos generales se presentaron diferencias según las variables sociodemográficas analizadas tanto en los indicadores de autopercepción, como en aquellos clínicamente observables. Una buena parte de la población adulta mayor no tiene prótesis a pesar de que la necesita y en otros casos requieren cambios en las prótesis superior e inferior para mejorar su funcionalidad y estética. Se evidencian algunas dificultades de higiene oral en la población analizada y se evidenciaron algunas lesiones de la mucosa oral que requieren supervisión por especialista en estomatología y medicina oral. A pesar de la población de adultos mayores no es prioritaria para el sistema de salud, especialmente cuando de atención odontológica se refiere, investigaciones como esta justifican la necesidad de brindar a este tipo de personas medios que les permita afrontar el proceso de envejecimiento por medio de una atención oportuna, segura y pertinente para contribuir al incremento del nivel de bienestar y calidad de vida en estos ciudadanos.

Los resultados encontrados evidencian la situación de vulnerabilidad social de la población adulta mayor. La literatura ha llamado la atención sobre como grupos sociales con altas necesidades de atención en salud, son aquellos grupos que menos cuidados reciben. Esto se conoce como la ley del cuidado inverso (Hart, 1971; Jones, 2001). De igual forma, la paradoja de la inequidad de Frohlich, advierte la existencia de grupos de población que por sus características sociales o económicas, son especialmente vulnerables a evidenciar situaciones dedesigualdad o inequidad en sus indicadores de salud (Frohlich \& Potvin, 2008), y sus factores de riesgo, como en este caso la población adulta mayor, y los diferentes subgrupos que se generan por sus determinantes sociales, que contribuyen a que existan vulnerabilidades que se acumulan con el paso del tiempo (Astrom et al., 2011). La población adulta mayor tiene una carga importante de enfermedad oral como efecto de las desigualdades a las cuales han estado sometidos desde edades tempranas y que tiene implicaciones para priorizar políticas públicas en este grupo social (Griffin et al., 2012).

Un hallazgo importante de esta investigación tiene que ver con las diferencias encontradas en los indicadores con relación a características sociodemográficas. Por ejemplo, la pérdida dental aumenta a medida que aumenta la edad y a su vez dicha pérdida está relacionada con pertenecer a un estrato socioeconómico bajo, a tener estudios bajos o nulos, al igual que vivir en la zona rural y tener ocupaciones manuales. Lo anterior indica la presencia de un gradiente social, es decir la situación de salud oral de los adultos mayores se ve afectada por las características sociales que estos poseen (Tsakos et al., 2011).

Se destacan las diferencias encontradas en el promedio de dientes cariados según el centro de atención al que acude la población estudiada. Lo anterior supone que son las Unidades Hospitalarias las que tienen mayor oportunidad en el servicio y por consiguiente las que tienen mayor capacidad de respuesta para dar atención en comparación con los centros de salud. El promedio de dientes perdidos por caries es un indicador que expresa las desigualdades históricas en el acceso a los servicios de salud que han obstaculizado que mas personas lleguen a edades avanzadas con el mayor número de dientes presentes, los cuales tal y como se menciono anteriormente, comparten gradientes sociales importantes. Un estudio cualitativo previo realizado desde la perspectiva de los prestadores directamente involucrados en los servicios de salud mencionan que existen barreras estructurales de acceso a la atención en salud para estos adultos en Medellín relacionadas con la infraestructura de la red de atención publica y con el Sistema General de Seguridad Social en Salud del país (AgudeloSuárez et al.). Un estudio realizado en Estados Unidos, muestra como existen necesidades que no son resueltas en la población y que van determinadas por factores sociales, por la capacidad económica y por el nivel de aseguramiento en el sistema de salud, lo cual determina el perfil de utilización de los servicios odontológicos (Jang et al., 2014).

Un punto a explicar desde los resultados encontrados, tiene que ver con la apropiación del autocuidado de la salud oral en la que se involucran actividades tan sencillas como el cepillado y la seda dental. La caries dental y la enfermedad periodontal son enfermedades de gran impacto en la población adulta mayor. EI ENSAB IV, evidenció una experiencia de caries dental del $96,3 \%$ en el grupo poblacional de 65 a 74 años, con un índice COP promedio de 20,6 y se presenta enfermedad periodontal generalizada en más del $90 \%$ de las personas. De igual forma el 99 $\%$ presenta perdida dental parcial y un $54 \%$ presenta pérdida total de todos sus dientes (Ministerio de Salud 
y Protección Social, 2013-2014). Estas enfermedades son consideradas crónicas, que necesitan de un periodo para su desarrollo, lo que sugiere que esta población viene con antecedentes de posibles deficiencias en la higiene oral y en las prácticas de autocuidado (Moreno Castillo et al., 2012).

Llama la atención las diferencias encontradas por sexo en el componente de dientes cariados ya que son las mujeres las que tienen menos dientes cariados (con diferencias significativas con respecto a los hombres). Lo anterior puede explicarse desde la perpectiva de género, definido éste como el conjunto de roles sociales (Sen et al., 2005); según esto son las mujeres las que a traves de la historia han sido las cuidadoras del hogar y de las personas que tienen a cargo, en otros casos, son ellas las que buscan continuamente los servicios de salud y permanecen alerta sobre lo que pueda pasar en relación con su salud (Mendoza-Sassi et al., 2003). De ahí se desprende el hecho de que son atentidas en estadios iniciales y menos graves de la enfermedad oral, lo que se expresa para este caso como un mejor estado del componente oral, lo anterior se se ve reflejado en un mayor numero de dientes obturados en comparación con los hombres.

En cuanto a los indicadores de autopercepción analizados para la población adulta mayor, la mitad o más de los participantes en el estudio reportaron problemas y mal estado de salud oral e insatisfacción general con su estado de dientes y prótesis. Estos indicadores guardaron estrecha relación con el examen clínico, lo cual guarda coherencia con estudios que relacionan los indicadores subjetivos con los parámetros clínicos (Schutzhold et al., 2014). La literatura comenta la importancia de la utilización de los indicadores de salud oral autopercibida como predictores del comportamiento del individuo, de la utilización de los servicios de salud odontológicos y permiten la construcción social del problema (Reisine \& Bailit, 1980; Kiyak, 1993; Kaplan \& Baron-Epel, 2003).

Llama la atención al analizar el estado protésico de las personas al encontrar diferencias significativas por el sexo. Gran porcentaje de la población requiere cambio de sus prótesis, especialmente las mujeres. En cuanto a la higiene de las prótesis, una buena parte de los hombres y las mujeres no se retiran la prótesis para dormir (con diferencias significativas en el caso de la prótesis inferior). En cuanto a la limpieza de la mucosa son más los hombres que las mujeres que no la limpian (y con diferencias estadísticamente significativas por sexo en ambos casos, superior e inferior). Estos resultados coinciden con otro estudio local en pacientes adultos edentados atendidos en una Institución Docencia-Servicio de la ciudad (Moreno Castillo et al.). De igual forma, otros estudios encontraron que los adultos mayores no limpiaban sus protesis y además establece como punto a la discusión el hecho de que se debe considerar el tiempo de uso de aparatos protésicos, dado que tiende a prolongarse debido a la creencia que tienen dichas personas sobre la longevidad de tales aditamentos, lo que conlleva a no recambiar su aparatología por períodos que superan los diez años (de Castellucci Barbosa et al., 2008; Ribeiro et al., 2009).

Muy relacionado con lo anterior, radica en el estado de las prótesis dentales. El mal estado de las mismas puede generar deficiencias en la masticación, lo que redundaría en la generación de otras patologías incluso a nivel sistémico. Por ejemplo, el estudio de von Kretschmann San Martin et al. (2014) realizado en Chile, indicó que los pacientes portadores de prótesis totales señalan un bajo nivel de satisfacción con el uso de sus dentaduras completas, en especial durante la función masticatoria. Las prótesis en mal estado y desadaptadas pueden ocasionar la aparición de patologías en los tejidos blandos tal y como lo reporta Mujica et al. (2008), en el que además se evidenció en que la mala higiene estaba asociada a la presencia de dichas lesiones. Por otro lado, de la RosaGarcía et al. (2012) confirmaron que el uso de prótesis dentales es un factor de riesgo importante para el desarrollo de Candidiasis oral. En el presente estudio, los dos tipos de lesiones más frecuentes que presentaron en la mucosa oral para los hombres fueron la candidiasis y la leucoplasia, mientras que para las mujeres predominaron las ulceras y la candidiasis (se presentaron diferencias estadísticamente significativas para el caso de la leucoplasia según el sexo). Estos resultados van en consonancia con otros estudios donde se encontró baja prevalencia de estomatitis, pero que de igual forma se detectaron otras lesiones en pacientes adultos mayores como por ejemplo queilitis angular, úlceras, fibromas, incluso melanosis y leucoplasia (Espinoza et al., 2003).

Como fortalezas de este estudio comentar que se contó con una muestra suficiente de adultos mayores representativa de las Unidades Hospitalarias y Centros de Salud de la red prestadora de servicios de salud pública de la ciudad de Medellín lo cual permite visibilizar la situación de salud oral en un segmento 
importante de especial vulnerabilidad social. De igual manera el uso de instrumentos de autopercepción y clínicos permite el análisis desde indicadores objetivos y subjetivos trascendiendo la forma de análisis de los problemas de salud oral desde un punto de vista exclusivamente biomédico. Se realizó control de calidad en los cuestionarios, lo cual favorece su confiabilidad. No obstante, en la interpretación de los resultados se deben tener en cuenta las limitaciones del estudio. Conviene mencionar que la naturaleza transversal del estudio no permite establecer causalidad en las relaciones y asociaciones encontradas. Se sugiere ampliar este estudio a otros adultos mayores institucionalizados en la ciudad y aquellos vinculados a otros estratos sociales con el fin de identificar situaciones de desigualdad y complementar con análisis de factores contextuales, factores nutricionales y de riesgo cardiovascular que permitan analizar los determinantes de la salud oral de la población adulta mayor desde una perspectiva integral e integradora.

La realización del IV Estudio Nacional de Salud Bucal y su análisis desde la perspectiva del curso de vida o ciclo vital humano permite la identificación de los problemas y las necesidades de la población por grupos poblacionales como los adultos mayores (Ministerio de Salud y Protección Social, 2013-2014). De ahí que se requiera la incorporación de sistemas de vigilancia epidemiológica de los principales indicadores de salud oral en esta población e identificar los factores determinantes, desde la identificación de realidades locales diversas, el análisis de la situación en colectivos de especial vulnerabilidad y la realización de políticas y estrategias desde un abordaje multisectorial reconociendo el derecho a la salud oral de todos los ciudadanos.

\section{AGRADECIMIENTOS}

El grupo de investigación agradece al grupo de adultos mayores que participaron en el estudio y al grupo de profesionales y estudiantes que participaron en el trabajo de campo en la realización de encuestas y exámenes clínicos.

POSADA-LÓPEZ, A.; AGUDELO-SUÁREZ, A. A. \& MENESES-GÓMEZ, E. J. Oral health conditions and prosthetic status in elderly population assisted by the public hospital network of Medellin (Colombia). Int. J. Odontostomat., 10(1):161-171, 2016.

ABSTRACT: This study aimed to analyze oral health conditions and prosthetic status in elderly population assisted by the public hospital network in Medellin (Colombia). A cross sectional study was conducted by means a two-stage sampling in hospital units and health centers attached to this network. 342 adults aged 65 and over (58.2 \% women) participated and the information was collected through survey and clinical examination. Variables: Sociodemographic: age, sex, socioeconomic status, educational level, place of residence, social support (Duke-11). Oral Health: Self-perceived Oral health, presence of oral problems in the last month. Clinical indicators: DFMT Index, present teeth, state of dentures, oral mucosa problems. Univariate and bivariate analysis and calculation of chi-square tests were carried out to observe statistically significant differences among different variables. As main findings people $\geq 75$ years, men, people of socioeconomic and educational level lower, located in urban areas and with low social support tend to report poor self-perceived oral health. The average teeth in the population was $5.7( \pm 7.8)$, implying a 24.2 average DFMT $( \pm 5.9)$, differing according to demographic variables. Over $55 \%$ of men and women require lower denture change and $70 \%$ of them require change of upper prostheses. Main oral mucosa problems were observed: Candidiasis (12\%), ulcers (7\%), leukoplakia (7\%), lichen planus (1\%). As a conclusion poor oral health conditions was observed and they can be explained on the situation of social vulnerability faced o this population and barriers to preventive and curative oral health care.

KEY WORDS: oral health, dental prosthesis, oral hygiene, health of the elderly, dental care.

\section{REFERENCIAS BIBLIOGRÁFICAS}

Agudelo-Suárez, A. A.; Alzate-Urrea, S.; López-Vergel, F.; López-Orozco, C.; Espinosa-Herrera, E.; Posada-López, A. \& Meneses-Gómez, E. J. Barreras y facilitadores de acceso a los servicios de salud bucal para la población adulta mayor atendida en la red pública hospitalaria de Medellín, Colombia. Rev. Gerenc. Polit. Salud, 13(27):181-99, 2014.
Astrom, A. N.; Ekback, G.; Ordell, S. \& Unell, L. Social inequality in oral health-related quality-of-life, OHRQoL, at early older age: evidence from a prospective cohort study. Acta Odontol. Scand., 69(6):334-42, 2011.

Cardentey García, J.; Trujillo Otero, P. E.; Silva Contreras, A. M.; Sixto Iglesias, M. \& Crespo Palacios, C. L. Esta- 
do de salud bucal de la población geriátrica perteneciente a la Clínica Ormani Arenado. Rev. Cienc. Med., 15(2):7686, 2011.

Cardona Jiménez, J. L.; Villamil Gallego, M. M.; Henao Villa, E. \& Quintero Echeverri, A. El sentimiento de soledad en adultos. Medicina U. P. B., 32(1):9-19, 2013.

de Castellucci Barbosa, L.; Ferreira, M. R.; de Carvalho Calabrich, C. F.; Viana, A. C.; de Lemos, M. C. \& Lauria, R. A. Edentulous patients' knowledge of dental hygiene and care of prostheses. Gerodontology, 25(2):99-106, 2008.

de la Rosa-García, E.; Vera, H. F. L.; Espinoza, A. L. \& Mondragón, P. A. Factores de riesgo para candidosis asociada a prótesis bucal. Rev. Asoc. Dent. Mex., 69(6):266-70, 2012.

Departamento Administrativo Nacional de Estadística (DANE). Clasificación Internacional Uniforme de Ocupaciones adaptada para Colombia. Bogotá, Departamento Administrativo Nacional de Estadística (DANE), República de Colombia, 2005.

Espinoza, I.; Rojas, R.; Aranda, W. \& Gamonal, J. Prevalence of oral mucosal lesions in elderly people in Santiago, Chile. J. Oral Pathol. Med., 32(10):571-5, 2003.

Estrada Restrepo, A.; Cardona Arango, D.; Segura Cardona, A. M.; Ordóñez Molina, J.; Osorio Gómez, J. J. \& Chavarriaga Maya, L. M. Síntomas depresivos en adultos mayores institucionalizados y factores asociados. Univ. Psychol., 12(1):81-94, 2013.

Frohlich, K. L. \& Potvin, L. Transcending the known in public health practice: the inequality paradox: the population approach and vulnerable populations. Am. J. Public Health, 98(2):216-21, 2008.

Griffin, S. O.; Jones, J. A.; Brunson, D.; Griffin, P. M. \& Bailey, W. D. Burden of oral disease among older adults and implications for public health priorities. Am. J. Public Health, 102(3):411-8, 2012.

Hart, J. T. The inverse care law. Lancet, 1(7696):405-12, 1971.

Hobdell, M.; Petersen, P. E.; Clarkson, J. \& Johnson, N. Global goals for oral health 2020. Int. Dent. J., 53(5):285-8, 2003.

Jang, Y.; Yoon, H.; Park, N. S.; Chiriboga, D. A. \& Kim, M. T. Dental care utilization and unmet dental needs in older Korean Americans. J. Aging Health, 26(6):1047-59, 2014.

Jones, C. M. Capitation registration and social deprivation in England. An inverse 'dental' care law? Br. Dent. J., 190(4):203-6, 2001.
Kaplan, G. \& Baron-Epel, O. What lies behind the subjective evaluation of health status? Soc. Sci. Med., 56(8):166976, 2003.

Kiyak, H. A. Age and culture: influences on oral health behaviour. Int. Dent. J., 43(1):9-16, 1993.

Mendoza-Sassi, R.; Béria, J. U. \& Barros, A. J. D. Outpatient health service utilization and associated factors: a population-based study. Rev. Saude Publica, 37(3):3728, 2003.

Ministerio de la Protección Social. Resolución 3577 de 2006: Por la cual se adopta el Plan Nacional de Salud Bucal. Bogotá, Ministerio de la Protección Social, República de Colombia, 2006. Disponible en: https:// www.minsalud.gov.co/Normatividad Nuevo/ RESOLUCI\%C3\%93N\%203577\%20DE\%202006.pdf

Ministerio de la Protección Social. Decreto 3039 de 2007: Por el cual se adopta Plan Nacional de Salud Pública 2007- 2010. Bogotá, Ministerio de la Protección Social, República de Colombia, 2007. Disponible en: http:/ /www.ins.gov.co/normatividad/Normatividad/ DECRETO\%203039_2007\%20 PIan \% 20 Nacional\%20SP\%202007-2010.pdf

Ministerio de Salud y Protección Social. Resolución $n^{\circ}$ 008430 de 1993 (4 de octubre de 1993). Por la cual se establecen las Normas Científicas, Técnicas y Administrativas para la Investigación en Salud. Bogotá, Ministerio de Salud y Protección Social, República de CoIombia., 1993. Disponible en: https:// www.unisabana.edu.co/fileadmin/Documentos/ Investigacion/comite_de_etica/Res_8430_1993__Salud.pdf

Ministerio de Salud y Protección Social. IV Estudio Nacional de Salud Bucal. ENSAB IV: Para saber cómo estamos y saber qué hacemos. Bogotá, Ministerio de Salud y Protección Social, República de Colombia, 20132014. Disponible en: https://www.minsalud.gov.co/sites/ rid/Lists/BibliotecaDigital/RIDE/VS/PP/ENSAB-IVSituacion-Bucal-Actual.pdf

Ministerio de Salud y Protección Social. Plan Decenal de Salud Pública, PDSP, 2012-2021. Bogotá, Ministerio de Salud y Protección Social, República de Colombia, 2013. Disponible en: https:// w w w. $m$ i $n$ s a l u d. g o v. c o / Documentos $\% 20$ y $\% 20 \mathrm{Publicaciones/}$ Plan \% 20Decenal\%20-\%20Documento\% 20 en $\% 20$ consulta $\% 20$ para\%20aprobaci\%C3\%B3n.pdf

Misrachi L., C.; Sepulveda S., H. \& S. Lamadrid A., S. Situación protésica y conductas asociadas en adultos mayores de nivel socioeconómico medio-alto y bajo. Rev. Dent. Chile, 93(1):10-6, 2002. 
Montero, J.; Yarte, J. M.; Bravo, M. \& López-Valverde, A. Oral health-related quality of life of a consecutive sample of Spanish dental patients. Med. Oral Patol. Oral Cir. Bucal, 16(6):e810-5, 2011.

Moreno Castillo, J. A.; Montoya Sepúlveda, A. F.; Gómez Gil, D. P.; Arboleda Cadavid, A.; Zea Restrepo, F. J. \& Agudelo Suárez, A. A. Situación de salud bucal y estado protésico del paciente edentado bimaxilar que acude a la Facultad de Odontología de la Universidad de Antioquia: Un estudio piloto. Rev. Fac. Odontol. Univ. Antioq., 24(1):22-36, 2012.

Mujica, V.; Rivera, H. \& Carrero, M. Prevalence of oral soft tissue lesions in an elderly venezuelan population. Med. Oral Patol. Oral Cir. Bucal, 13(5):E270-4, 2008.

Organización Mundial de la Salud; Grupo Orgánico de Enfermedades No Transmisibles y Salud Mental; Departamento de Prevención de las Enfermedades No Transmisibles y Promoción de la Salud Envejecimiento y Ciclo Vital. Envejecimiento y Ciclo Vital. Envejecimiento activo: un marco político. Rev. Esp. Geriatr. Gerontol., 37(S2):74-105, 2002.

Petersen, P. E. The World Oral Health Report 2003: continuous improvement of oral health in the 21st century--the approach of the WHO Global Oral Health Programme. Community Dent. Oral Epidemiol., 31 Suppl. 1:3-23, 2003.

Petersen, P. E.; Bourgeois, D.; Ogawa, H.; Estupinan-Day, S. \& Ndiaye, $C$. The global burden of oral diseases and risks to oral health. Bull. World Health Organ., 83(9):661-9, 2005.

Population Reference Bureau. World Population Data Sheet 2014. Washington D. C., Population Reference Bureau, 2014. Disponible en: http://www.prb.org/pdf14/2014-worldpopulation-data-sheet_eng.pdf

Profamilia. Encuesta Nacional de Demografía y Salud: ENDS Bogotá, Profamilia, 2010. Disponible en: http:// profamilia.org.co/wp-content/uploads/2015/05/ends2010.pdf

Reisine, S. T. \& Bailit, H. L. Clinical oral health status and adult perceptions of oral health. Soc. Sci. Med. Med. Psychol. Med. Sociol., 14A(6):597-605, 1980.

Ribeiro, D. G.; Pavarina, A. C.; Giampaolo, E. T.; Machado, A. L.; Jorge, J. H. \& Garcia, P. P. Effect of oral hygiene education and motivation on removable partial denture wearers: longitudinal study. Gerodontology, 26(2):150-6, 2009.

Schutzhold, S.; Holtfreter, B.; Schiffner, U.; Hoffmann, T.; Kocher, T. \& Micheelis, W. Clinical factors and selfperceived oral health. Eur. J. Oral Sci., 122(2):134-41, 2014.
Sen, G.; George, A. \& Östlin, P. Incorporar la perspectiva de género en la equidad en salud: un análisis de la investigación y las políticas. Publicación Ocasional No. 14. Washington D.C., Organización Panamericana de la Salud, Harvard Center for Population and Development Studies, 2005

Sheiham, A. Oral health, general health and quality of life. Bull. World Health Organ., 83(9):644, 2005.

Tsakos, G.; Demakakos, P.; Breeze, E. \& Watt, R. G. Social gradients in oral health in older adults: findings from the English longitudinal survey of aging. Am. J. Public Health, 101(10):1892-9, 2011.

von Kretschmann San Martin, D.; Torres Varela, A.; Sierra Fuentes, M.; del Pozo Bassi, J.; Quiroga Aravena, R. \& R. Quiroga del Pozo, R. Rendimiento masticatorio y nivel de satisfacción de pacientes tratados con prótesis totales en la Universidad Mayor. Rev. Clin. Periodoncia Implantol. Rehabil. Oral, 8(1):17-23, 2015.

Watt, R. G. From victim blaming to upstream action: tackling the social determinants of oral health inequalities. Community Dent. Oral Epidemiol., 35(1):1-11, 2007.

World Health Organization. Oral Health Surveys. Basic Methods. 4th ed. Geneva, World Health Organization, 1997. Disponible en: http://www.paho.org/hq/ dmdocuments/2009/OH_st_Esurv

World Medical Association General Assembly. World Medical Association Declaration of Helsinki: ethical principles for medical research involving human subjects. J. Int Bioethique, 15(1):124-9, 2004

Dirección para Correspondencia:

Adriana Posada López

Facultad de Odontología

Universidad de Antioquia

Calle 70 No. 52-21

Medellín

COLOMBIA

Email: adriposalo@gmail.com

Recibido : 20-05-2015

Aceptado: 13-04-2016 